\title{
The modulation of somatosensory resonance by psychopathic traits and empathy
}

\section{Louis-Alexandre Marcoux ${ }^{1,2}$, Pierre-Emmanuel Michon ${ }^{3}$, Julien I. A. Voisin ${ }^{3,4}$, Sophie Lemelin ${ }^{1,2}$, Etienne Vachon-Presseau ${ }^{1,3}$ and Philip L. Jackson ${ }^{1,2,3 *}$}

1 École de Psychologie, Université Laval, Québec, QC, Canada

${ }^{2}$ Centre de Recherche de I'Institut Universitaire en Santé Mentale, Québec, QC, Canada

${ }^{3}$ Centre Interdisciplinaire de Recherche en Réadaptation et Intégration Sociale, Québec, QC, Canada

${ }^{4}$ Département de Réadaptation, Université Laval, Québec, QC, Canada

\section{Edited by:}

Bernadette M. Fitzgibbon, Monash

University, Australia

Reviewed by:

Alessio Avenanti, University of

Bologna, Italy

Daniela M. Pfabigan, University of

Vienna, Austria

*Correspondence:

Philip L. Jackson, École de

Psychologie, Pavillon

Félix-Antoine-Savard, 2325, rue des Bibliothèques, Université Laval,

Québec, OC GIV OA6, Canada

e-mail:philip.jackson@psy.ulaval.ca
A large number of neuroimaging studies have shown neural overlaps between first-hand experiences of pain and the perception of pain in others. This shared neural representation of vicarious pain is thought to involve both affective and sensorimotor systems. A number of individual factors are thought to modulate the cerebral response to other's pain. The goal of this study was to investigate the impact of psychopathic traits on the relation between sensorimotor resonance to other's pain and self-reported empathy. Our group has previously shown that a steady-state response to non-painful stimulation is modulated by the observation of other people's bodily pain. This change in somatosensory response was interpreted as a form of somatosensory gating (SG). Here, using the same technique, SG was compared between two groups of 15 young adult males: one scoring very high on a self-reported measure of psychopathic traits [60.8 \pm 4.98 ; Levenson's Self-Report Psychopathy Scale (LSRP)] and one scoring very low $(42.7 \pm 2.94)$. The results showed a significantly greater reduction of SG to pain observation for the high psychopathic traits group compared to the low psychopathic traits group. SG to pain observation was positively correlated with affective and interpersonal facet of psychopathy in the whole sample. The high psychopathic traits group also reported lower empathic concern (EC) scores than the low psychopathic traits group. Importantly, primary psychopathy, as assessed by the LSRP, mediated the relation between EC and SG to pain observation. Together, these results suggest that increase somatosensory resonance to other's pain is not exclusively explained by trait empathy and may be linked to other personality dimensions, such as psychopathic traits.

Keywords: pain perception, psychopathic traits, somatosensory resonance, shared representations, empathy

\section{INTRODUCTION}

Does vicariously experiencing someone else's pain help us understand and care about the distress this person might be feeling? Over the last decade, a large number of studies in healthy and clinical populations have used the representation of other people's pain as a means to investigate the different dimensions of empathy. The construct of empathy can be defined as the capacity to be in tuned with the affective experience of someone else. It involves, beyond a cognitive effort to understand and imagine someone else's state, a disposition to emotionally identify with other's feeling and to share their affective experience (Decety and Jackson, 2004; Kernberg, 2012a). Accordingly, this suggests that, at the brain level, multimodal neural networks are at play during empathic response. Early neuroimaging studies on pain observation have revealed a considerable overlap between cerebral regions involved in the direct experience of pain and its perception in others (e.g., Morrison et al., 2004; Singer et al., 2004; Jackson et al., 2005), suggesting the existence of a neuronal pathway implicated in the elaboration of representations that reflect our own responses to pain to understand how the pain of others feels (see Jackson et al., 2006; Lamm et al., 2011 for reviews). This shared neural representations between the perception of pain in self and other has been interpreted as the result of an automatic resonance mechanism (Jackson et al., 2006) that can be best described as the lower-level of a vicarious pain response on which higher order process operate to develop empathy (Han et al., 2009; Vachon-Presseau et al., 2011).

From initial clinical descriptions to contemporary taxonomies, psychopathy has been prototypically associated with severe emotional disturbances and empathy breakdown (Cleckley, 1941; Lilienfeld and Andrews, 1996; Hare, 2003). This personality disorder is better understood as a constellation of personality traits that encompass affective and interpersonal qualities along with behaviors reflecting a socially deviant lifestyle (Hare, 2003). Primary psychopathy has been designated as the heritable traits of emotional detachment commonly reported as a lack of compassion and guilt, callous misuse of others for personal gain and failure to form close interpersonal attachment (Levenson et al., 1995; Poythress and Skeem, 2006). Secondary psychopathy usually refers to poor behavioral control, hostility and antisociality 
(Levenson et al., 1995). The majority of research on psychopathy has focused on samples of incarcerated male offenders, which has led to some pending interrogations about the generalizability of these results to community samples (Hall et al., 2004). Still, studies in non-incarcerated samples have gained in popularity, as the dimensional approach to personality disorders has obtained support from both clinical and research fields (for a review on the clinical perspective see Kernberg, 2012b).

Several transcranial magnetic stimulation (TMS; e.g., Avenanti et al., 2005), somatosensory-evoked potentials (SEP; e.g., Bufalari et al., 2007; Martínez-Jauand et al., 2012), magnetoencephalography (e.g., Cheng et al., 2008), functional magnetic resonance imaging (fMRI; e.g., Lamm et al., 2007; Saarela et al., 2007; Han et al., 2009) and somatosensory steady-state response (SSSR; e.g., Voisin et al., 2011a) studies have shown that brain regions processing the sensory dimension of first hand pain (i.e., somatosensory cortices) are also modulated by the observation of visual stimuli depicting body limbs in pain (Voisin et al., 2011a), painful facial expressions (Saarela et al., 2007), and even psychological painful scenarios (e.g., social rejection in Kross et al., 2012). Some studies have also demonstrated that this resonance mechanism can be modulated by individual factors such as state-reactivity (Avenanti et al., 2009), trait empathy (Avenanti et al., 2009; Vachon-Presseau et al., 2011) and callous-unemotional traits (Fecteau et al., 2008). The study of Fecteau et al. (2008), in which a community sample of men was exposed to visual stimuli depicting hands in painful and non-painful scenarios, was the first to show a positive correlation between suppression of motor evoked potentials (MEPs) and the score of their participants on the Coldheartedness subscale of the psychopathic personality inventory (PPI; Lilienfeld and Andrews, 1996). This result seemed counter-intuitive because increase sensorimotor resonance to the pain of others had been positively associated with self-reported empathy (Avenanti et al., 2009). However, it was also suggested that this automatic neural response could trigger distress (Decety, 2011) and threat related networks (Ibáñez et al., 2011), therefore advocating for an alternative or concomitant view to automatic pain resonance that simply implies arousal. This would also support the view that regulation processes of sensorimotor responses are required in order to respond empathically to the pain of others (Han et al., 2009). Together, these results suggest that sensorimotor resonance to the pain of others is not a direct path to empathy and further investigation on the role of psychopathic traits could be useful to better understand this relationship.

One question arising is how psychopathic traits influence the somatosensory resonance mechanisms involved in the perception of pain in others. To date, only one study has investigated the sensorimotor resonance to other's pain in a community sample of men with psychopathic traits (Fecteau et al., 2008). Although this TMS study has revealed intriguing and initially counterintuitive findings, it has mainly focused on the motor aspect of resonance. Previous studies have shown that seeing pain in others reduces somatosensory steady-state response (SSSR) to a nonpainful stimulation (Voisin et al., 2011a) and that this reduction is specific to the frequency of the mechanical stimulation, reinforcing the idea that the modulation in SSSR reflects the inhibition (gating) of somatosensory activity by attention (Mayer et al.,
2009). In order to gain understanding on the relationship between psychopathic traits and sensory resonance, we measured SSSR of participants exposed to clips depicting pain-evoking or neutral situations.

The aim of the present study was to investigate the somatosensory aspect of the resonance to other's pain in two groups of men selected from a large community: one group scoring very high and one group very low on a psychopathic traits measure [Levenson's Self-Report Psychopathy Scale (LSRP), Levenson et al., 1995]. Another objective of this study was to examine the relationship between the somatosensory response, self-reported empathy, and psychopathy. We used the modulation of the somatosensory response to a mechanical stimulation as a function of the visual stimuli depicting different levels of bodily pain (Voisin et al., 2011a) as a measure of somatosensory gating (SG). This response was subsequently compared with: (1) vicarious pain ratings, (2) the scores on a measure of trait-empathy [Interpersonal Reactivity Index (IRI), Davis, 1980] and (3) the scores on the LSRP (Levenson et al., 1995). We first expected to find lower scores on the affective subscale of the IRI in high psychopathic traits males compared to the low psychopathic traits ones. Taking into account that both the hypotheses of sensorimotor resonance mechanisms (Bufalari et al., 2007; Lamm et al., 2007) and arousal (Decety, 2011) might be at play during pain empathy, we also expected that participants with high psychopathic traits would have a greater SG to pain observation compared to individuals with low psychopathic traits. Finally, according to Fecteau et al. (2008) we posited that SG would be positively correlated with the affective and the interpersonal facets of psychopathy.

\section{MATERIALS AND METHODS PARTICIPANTS}

Only males were invited to participate to this experiment because the prevalence of psychopathy in women is much lower than in men (e.g., Salekin et al., 1997; Jackson et al., 2002). One hundred and sixty four undergraduate right-handed male students were recruited across different Faculties of Université Laval, Québec, and asked to complete the LSRP (Levenson et al., 1995; see description below) in class. From this initial sample, and based on the distribution of the LSRP_Total scores, two sub-groups were invited to participate to an EEG protocol: 15 participants in the upper third (LSRP_High), and 15 participants in the lower third (LSRP_Low) (see Table 1 for detailed characteristics

Table 1 | Mean age and scores on self-reports of psychopathy.

\begin{tabular}{llllll}
\hline Groups & $\boldsymbol{N}$ & Age & LSRP_Total & PP1 & PP2 \\
\cline { 3 - 6 } & & $\boldsymbol{X}(\boldsymbol{S D})$ & $\boldsymbol{X}(\boldsymbol{S} \boldsymbol{D})$ & $\boldsymbol{X}(\boldsymbol{S D})$ & $\boldsymbol{X}(\boldsymbol{S D})$ \\
\hline Whole sample & 164 & $22.2(2.75)$ & $50.9(6.31)$ & $34.2(5.6)$ & $18.1(3.2)$ \\
LSRP_Low & 15 & $23.7(2.9)$ & $42.7(2.94)^{* * *}$ & $27.7(4.7)^{* * *}$ & $17.1(2.9)^{* *}$ \\
LSRP_High & 15 & $22.3(1.44)$ & $60.8(4.98)^{* * *}$ & $38.8(4.3)^{* * *}$ & $20.0(4.3)^{* *}$
\end{tabular}

${ }^{* *} p<0.01,{ }^{* * *} p<0.001$. PP1, primary psychopathy subscale; PP2, secondary psychopathy subscale. 
of the sample). The LSRP_High total scores $(60.8 \pm 4.98)$ were significantly higher than the LSRP_Low scores $[42.7 \pm 2.94$; $\left.t_{(29)}=12.12, p<0.001\right]$. Participants reported having no history of neurological, pain-related, or psychiatric disorders, were not taking any medication, and had normal or corrected-tonormal vision. The participants received monetary compensation for their travel expenses to the laboratory and they each gave written informed consent. The study was approved by the Ethics Committees of the research center (CIRRIS-IRDPQ) and Université Laval.

\section{MEASURES AND MATERIALS Questionnaires}

The LSRP (Levenson et al., 1995) is a 26-item self-reported measure of psychopathic traits developed for use in community samples. The LSRP assess primary and secondary psychopathy, two factors of the most predominant psychopathic measure, the Hare Psychopathy Checklist (PCL-R; Hare, 2003). Each item consists in a statement that the participant endorses on a 4-point Likert-type scale ( $1=$ disagree strongly to $4=$ agree strongly). The primary psychopathy subscale (PP1) consists in 16 items measuring an inclination to lie, a lack of remorse, callousness, and manipulativeness. The secondary psychopathy subscale (PP2) consists in 10 items measuring impulsivity, frustration tolerance, quick-temperedness, and lack of long-term goals.

The Davis' IRI (Davis, 1980) is a 28-item self-report instrument that assesses trait empathy, that is, one's own reactions to the observation of another's experiences. Each item is rated on a scale ranging from 1 (does not describe me well) to 5 (describes me very well). The IRI is composed of four subscales thought to reflect the affective and cognitive aspects of empathy: Empathic Concern
(EC) and Personal Distress (PD), Fantasy (FS) and Perspective Taking (PT). The EC subscale measures experienced feelings of sympathy and compassion for others in distress. The PD measures self-oriented feelings of anxiety and distress in response to tense interpersonal situations. The FS scale measures the tendency to project oneself into fictional situations. The PT subscale measures the tendency to adopt the psychological point of view of others.

The situational pain questionnaire (SPQ; Clark and Yang, 1983) was used in order to evaluate how participants estimated their own sensitivity to pain. The discrimination scores $\mathrm{P}(\mathrm{A})$, indicate the extent to which subjects are able to differentiate painful scenarios from neutral, while the response bias scores $\mathrm{B}$, indicate the degree to which the situations are considered painful (for details on the method see Danziger et al., 2006). The questionnaire consists in 15 events that are considered to be relatively painful and 15 non-painful events. Items are rated by using a numerical scale ranging from 1 (not noticeable) to 10 (worst possible pain).

\section{Visual stimuli}

Stimuli consisted in a series of 30-color pseudo-dynamic pictures depicting hands of male and female adults in three different conditions: Painful, Non-Painful, and Neutral situations. Specifically, each stimulus involved a sequence of three visual static pictures presented in a short sequence $(750 \mathrm{~ms}+250+1500=2500 \mathrm{~ms})$ to create the illusion of a movement (similar to the task described in Decety et al., 2009; see Figure 1). Different types of pain (mechanical and thermal) inflicted to the hands were displayed. The No Pain stimuli showed hands in visually similar situations as in the Pain condition but without the painful consequence [i.e., the 3rd frame differed; e.g., a knife on the finger (Pain) vs.

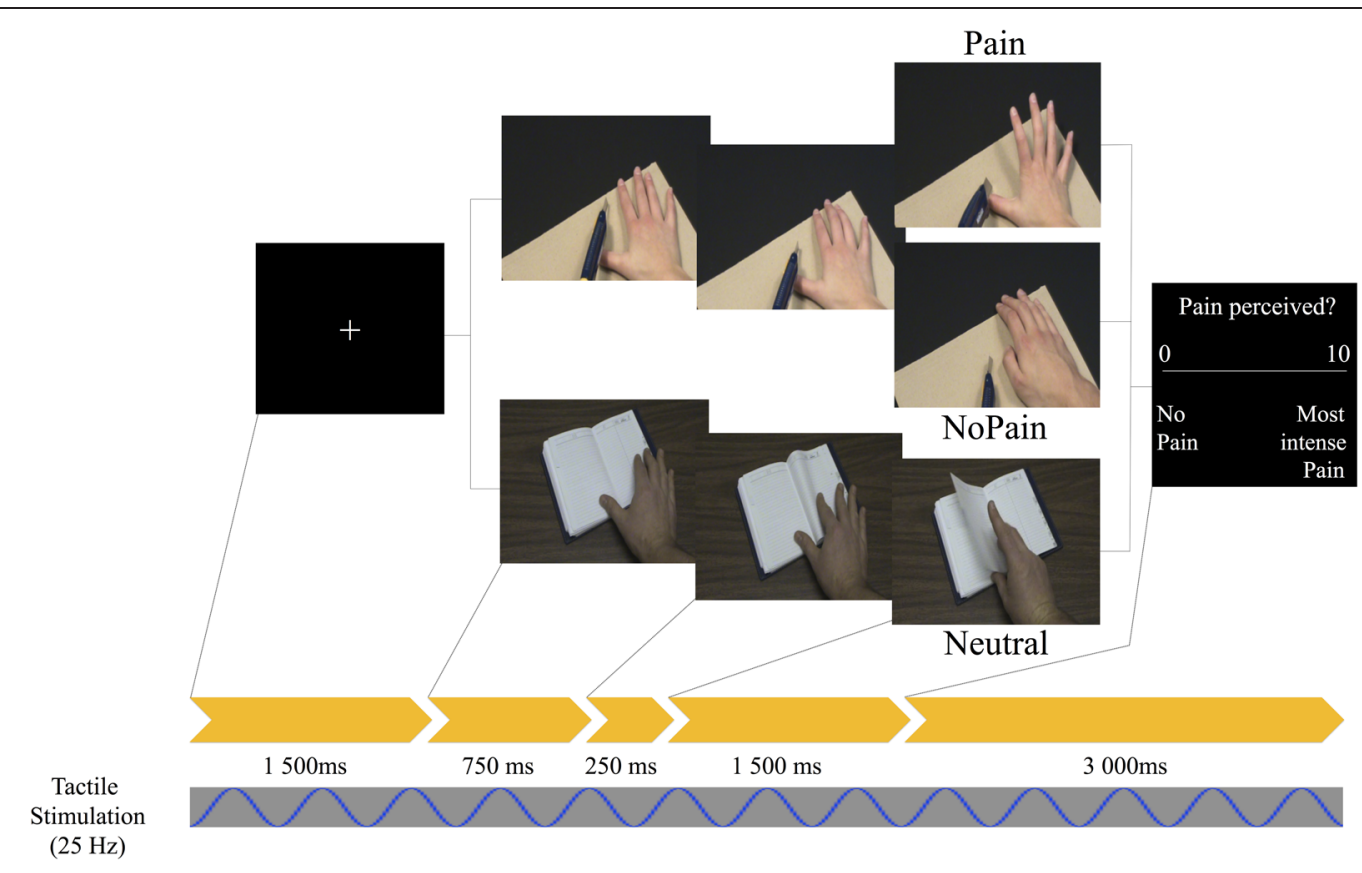

FIGURE 1 | Schematic of the experimental design depicting one trial. Timing in ms (below yellow arrows) corresponds to the duration of each picture. A light repetitive stimulation at a frequency of $25 \mathrm{~Hz}$ was continuously applied to the palm of the right hand throughout data acquisition. 
a knife of the board (No Pain)]. Neutral stimuli showed hands in visually different situations devoid of any of the nociceptive elements found in the other two conditions (e.g., a hand grasping a set of keys or a tissue). We used a neutral condition to assess the possible priming effect of the nociceptive elements already found on the first picture of the Painful and Non-Painful conditions. The hands were shown from a maximum angle of $45^{\circ}$ from the perspective of the observer, and all pictures were edited to show hands of same size and from approximately the same distance.

\section{Tactile steady-state stimulation}

Non-painful light repetitive $(25-\mathrm{Hz})$ mechanical stimulations were continuously applied to the palm of the right hand using a custom-made vibrotactile stimulator similar to the one used in Voisin et al. (2011a,b). Compared to the previous stimulator, which targeted the ventral portion of the right index distal phalange, the one used in the current study stimulated the whole palm of the right hand.

\section{EEG}

EEG activity was acquired via $124+4 \mathrm{Ag} / \mathrm{AgCl}$ electrodes contacting the scalp surface by way of saline-soaked sponges (HCGSN, Electrical Geodesic Inc., Oregon). The amplifier system used for EEG recordings was an EGI GES250 system (Electrical Geodesic Inc., Oregon). The sampling rate was $500-\mathrm{Hz}$, with acquisition reference at the vertex. Electrodes impedances were kept below $50 \mathrm{k} \Omega$.

\section{Electromyographic activity}

In order to ensure that the modulation in SG was not due to muscle contraction of the right hand, electromyographic activity (EMG) was recorded in all participants using $\mathrm{Ag}-\mathrm{AgCl}$ surface electrodes placed in bipolar configuration over the First Dorsal Interosseus (FDI) muscle. EMG was amplified and band pass filtered $(20-1000 \mathrm{~Hz})$. The Acknowledge software (Biopac System) was used to acquire surface EMG and events code. Online visual inspection of the EMG output and inter-block feedback to participants ensured that this muscle stayed relaxed during EEG data acquisition and that the energy contained in the $25-\mathrm{Hz}$ band frequency was produced by the stimulation.

\section{PROCEDURE}

Participants took part in a $60 \mathrm{~min}$ EEG session. They were seated in an armchair with their right arm on an arm-rest while watching a $20^{\prime \prime}(\sim 48 \mathrm{~cm})$ LCD monitor positioned approximately at $85 \mathrm{~cm}$. Stimuli were presented with a computer running the E-Prime software (Version 2.0, Psychology Software Tools, Inc.) to control the timing of the stimuli as well as the generation of event codes. Each trial began by a fixation cross $(2500 \mathrm{~ms})$, followed by a sequence of three static pictures (total time $2500 \mathrm{~ms}$ ) successively presented, ending with visual rating scale (3000 ms) ranging from 0 (no pain) to 10 (worst pain possible) (see Figure 1). Subjects were told to refrain from blinking and performing head and jaw movements as much as possible during the presentation of fixation crosses and stimuli. After each scenario, participants were instructed to use the visual rating scale and verbally evaluate the level of pain that individuals would feel in each scenario via an intercom system as participants were seated in an audiometric room (Genieaudio Inc., Toronto). The experimental session consisted of six blocks of 30 trials lasting approximately 5 min each. The conditions were randomized and counter-balanced within each of the six blocks. Several practice trials were run prior to the experiment using other picture than those selected for the test trials. After the six experimental blocs, participants were asked to fill self-reported trait-empathy (IRI) and pain sensitivity (SPQ) questionnaires.

\section{EEG DATA PREPROCESSING}

All preprocessing was performed with the ELAB software developed at Centre de recherche en réadaptation et intégration sociale (CIRRIS) (Voisin et al., 2011a,b). ELAB is a series of Matlab routines allowing the control of the ELAN-Pack software developed at INSERM Brain Dynamics and Cognition team of the Lyon Neuroscience Research Center (Aguera et al., 2011). Raw data was first parsed into event, and indexed according to the type of the stimuli. Two faulty electrodes caused unreliable signal across all subjects and were removed from the analysis [electrodes 83 and 114 in the EGI system (HCGSN) corresponding to T10 and $\mathrm{O} 2$ in the 10-20 systems]. Then, a first rejection criterion was applied on the basis of any rating for a Painful stimulus $<1$, for a Neutral or Non-Painful stimulus $>1$ led to the rejection of the related-event, to ensure that further analyses would be made only on task-relevant data. Inspection of the data distribution enabled the selection of a series of criteria meant to detect blinks, muscle activity, and fast baseline shift. They were set to reject any sample that fell within $100 \mathrm{~ms}$ of one of these events: (1) the scalp potential exhibited variation over $200 \mu \mathrm{V}$ within a $200 \mathrm{~ms}$ time window in the same electrode channel; (2) the energy content was more than $500 \mu \mathrm{V}^{2}$ in the $60-100 \mathrm{~Hz}$ band in the same electrode channel; (3) the scalp potential exhibited variation over $50 \mu \mathrm{V}$ within a $10 \mathrm{~ms}$ time window in the same electrode channel; (4) the energy content was more than $1500 \mu \mathrm{V}^{2}$ in the $23-27 \mathrm{~Hz}$ band in the same electrode channel. The remaining data consisted of $77 \%$ of the original set. This remaining signal was submitted to a spherical spline interpolation process (Perrin et al., 1989), using Tikhonov regularization in order to reduce sensitivity to noise (Babiloni et al., 1998). This procedure allows the reconstruction of the signal of a noisy electrode based on the signal of the noise-free electrodes. Notably, this process poses a specific challenge as the rejected samples can be broadly distributed across time and electrodes so that a proper reconstruction has either to reject all samples each time a faulty electrode is found, or to reject all electrodes that included at least one rejected sample. Thus, any fixed method would have led to rejecting a large portion of the data. ELAB software allowed circumventing this problem by selecting, automatically for each trial, the set of electrodes that should enter the interpolation process so as to maximize the number of valid samples used. In the present experiment, the best solutions used a mean of $70 \%$ of the original samples (intersubject variability 48-93\%) to reconstruct the signal. More precisely, the interpolation process was based on average on $77 \%$ of the 124 electrodes positioned on the scalp (intersubject variability 47-97\%) and on average, 91\% of the time bins (intersubject variability $68-99 \%)$. Once the signal was split-transformed, it 
was convoluted with complex Gaussian Morlet's wavelets (TallonBaudry and Bertrand, 1999) intended to extract the energy in the $25 \mathrm{~Hz}$ range (omega, 24-26; sigma, 3.6), representing the energy band in which the cortical response to the somatosensory stimulation used in the current study should be condensed. Mean $25 \mathrm{~Hz}$ range energy during the fixation cross $(1000 \mathrm{~ms}$ before stimulus onset) was then computed, and any trial in which the baseline mean energy dispersion was over two standard deviation from the whole bloc mean energy was rejected (an average of one trial was rejected per subject, max rejection was two trials). No subject was rejected from analysis.

\section{STATISTICAL ANALYSES \\ Behavioral data}

Differences on mean pain ratings between conditions and groups were computed using a 3 (Conditions: Pain vs. NoPain vs. Neutral) $\times 2$ (Groups: LSRP_high vs. LSRP_low) repeated measures analysis of variance (ANOVA). The relation between pain ratings and psychopathy scores (LSRPtotal, PP1, and PP2) were explored with Pearson correlations. In order to assess between group differences on the independent subscales of self-reported empathy (IRI), four independent sample $t$-tests were realized. Pearson correlations were then used to determine the relationship between empathy and psychopathy scores. Finally, group differences on pain sensitivity discrimination $\mathrm{P}(\mathrm{A})$ and bias scores (B) of the SPQ were tested with two independent sample $t$-tests.

\section{EEG}

A similar procedure as in Voisin et al. (2011a) was used to analyze the SSSR. First, epochs in all three conditions were averaged to delineate the regions of interest (ROI) for each group. Subtraction maps were then created by subtracting the baseline period $(-1000: 0 \mathrm{~ms}$, the cross duration) from the first two pictures period $(0: 1000 \mathrm{~ms})$. This procedure allows the visual identification of the electrodes in which SG was showing the greatest modulation during the first two pictures in comparison to baseline (fixation cross), for all conditions. Note that the maps were created from (1000 ms) time bins and statistical analyses were then all realized with more circumscribed 200ms time bins to increase accuracy. This initial analysis identified the following ROI electrodes [parietal electrodes 66, 67, and 71 in the EGI system (HCGSN) corresponding to P3 in the 10-20 systems] on which the remaining of the analyses was done.

Prior to test the non-specific initial gating (i.e., not imputable to the observation of pain), defined as the mean energy $(\mathrm{mA} / \mathrm{m} 3)$ difference between Fixation Cross Baseline (-200:0 ms) and Gating period (600:800 ms) (see Voisin et al., 2011a), Cross Baseline stability was verified using a 3 (Conditions: Pain vs. NoPain vs. Neutral) $\times 2$ (Groups: LSRP_high vs. LSRP_low) repeated measures ANOVA. To investigate initial gating effect, mean energy during Gating period $(600: 800 \mathrm{~ms})$ and Cross Baseline (-200:0 ms) were compared for each condition using simple $t$-tests against $\mathrm{H}_{0}$ (i.e., absence of gating). Second, pain anticipation $[($ Pain $=$ Nopain $)>$ Neutral] was tested by comparing mean energy ratios between the three experimental conditions during the Gating period (600:800 ms) with a one-way repeated measures ANOVA [Gating period $\times$ Conditions (3: Pain vs.
NoPain vs. Neutral)]. Third, Pain Gating was assessed using ratios [(Second Picture Baseline - 3rd Picture Pain Gating)/Second Picture Baseline] by comparing painful and non-painful conditions for each participant in order to verify the specific modulation imputable to the onset of painful conditions using a 2 (Conditions: Pain vs. NoPain) $\times 2$ (Groups: LSRP High vs. LSRP Low) repeated measures ANOVA. The 3rd Picture Gating period (1100:1700 ms) was divided in three $(200 \mathrm{~ms})$ time bins. Separated analysis was performed on each time bin. All the analyses were done with an alpha level set at 0.05 and corrected with Bonferroni procedure for multiple comparisons.

\section{Mediation analysis}

As sensorimotor resonance was previously found to be positively associated with scores on Coldheartedness subscale (Fecteau et al., 2008), which reflect a lack of empathy and sensibility toward others, and conversely positively correlated with trait-empathy (Avenanti et al., 2009), we sought to explore the indirect effect of primary psychopathy on the relationship between self-reported empathy and SG to pain. This was tested using the bootstrapping method developed by Preacher and Hayes (2004, 2008; see Simple Mediator model). This non-parametric method overcomes limitations of the Baron and Kenny's (1986) causal steps and Sobel's test that are conservative and not likely to detect indirect effects in smaller samples. Moreover, this method has the benefit of not assuming normality of the sampling distribution of the indirect effect and allows testing of mediating effect (Preacher and Hayes, 2004). The SPSS macro developed by Preacher and Hayes (2008) provides the strength of direct effects of independent and mediating variables. Preacher and Hayes (2004) also stated that it is possible to find a significant indirect effect even if there is no evidence of a significant total effect (path c, see Figure 7). Pointestimate of the indirect effect and $95 \%$ bias corrected confidence intervals (BC) were computed based on a 5000 bootstrap resample. In order to conclude for the presence of a mediating effect, the 95\% BC confidence interval must not include zero, thus suggesting that the value of the indirect effect is significantly different from zero. Note that the relatively small sample in the current study suggests caution in drawing inference from the mediation analysis.

\section{RESULTS}

\section{BEHAVIORAL RESULTS}

Analyses performed on pain intensity ratings confirmed the expected significant effect for the main effects of Condition $\left[F_{(1,28)}=160.7, p<0.001\right]$ whereas no significant effect was observed for Group $\left[F_{(1,28)}=0.21, p=0.657\right]$ nor their interaction $\left[F_{(1,28)}=1.19, p=0.283\right]$. Post-hoc pair comparisons showed that mean pain ratings for painful scenarios $(4.9 \pm 0.362)$ differed significantly from non-painful $(0.002 \pm 0.001 ; p<$ $0.001)$ and neutral scenarios $(0.03 \pm 0.021 ; p<0.001)$ whereas no difference has been found between the latter two $(p=0.143)$. As illustrated in Figure 2A, between-group analyses showed no significant differences for the mean ratings in the pain condition (LSRP_High: $5.1 \pm 0.441$; LSRP_Low: $4.5 \pm 0.473$ ). To compare the differences between LSRP_Low and LSRP_High participants on trait empathy, independent $t$-test on each IRI subscale 
were used and revealed no significant between-groups difference on three of the four subscales [PT: $t_{(29)}=2.3, p=0.142 ; \mathrm{F}$ : $t_{(29)}=0.562, p=0.47$; $\left.: t_{(29)}=0.962, p=0.344\right]$. Figure 2B shows the only subscale (EC) for which a significant difference between both groups was found [LSRP_Low: 19.6 \pm 3.7 ; LSRP_High: $\left.14.1 \pm 4.9 ; t_{(29)}=10.9, p=0.003\right]$. Over all participants, the correlations showed a significant negative relationship between the EC subscale and the LSRP_total score $(r=-0.561$,

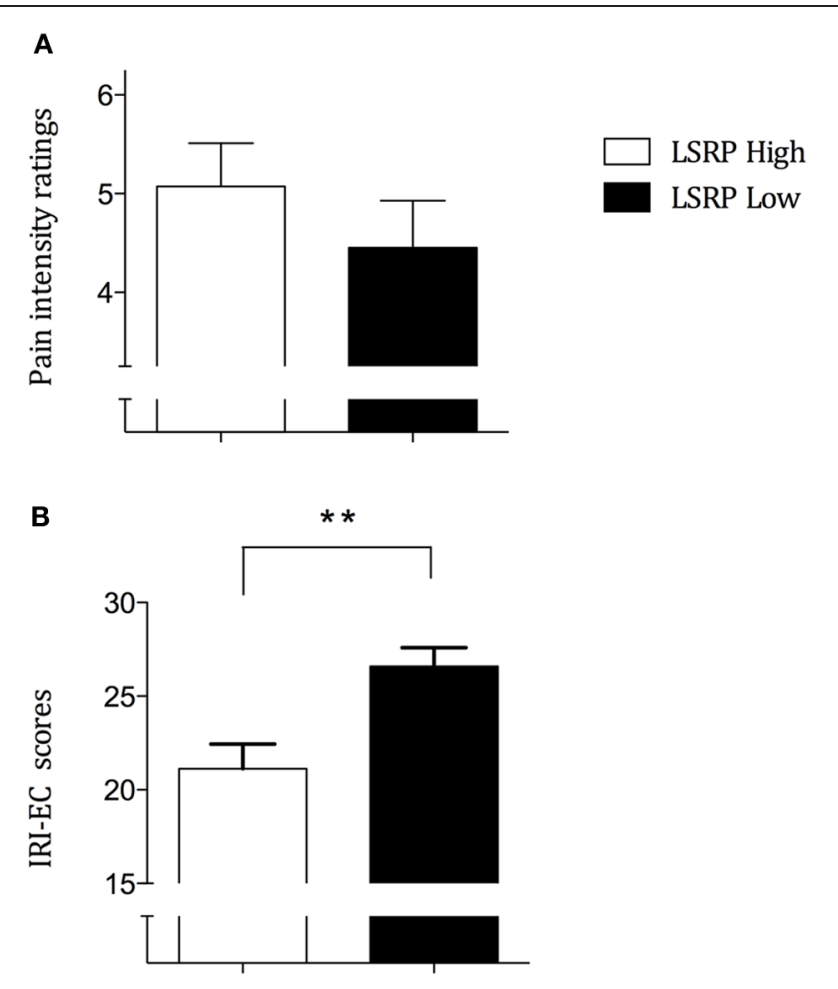

Groups

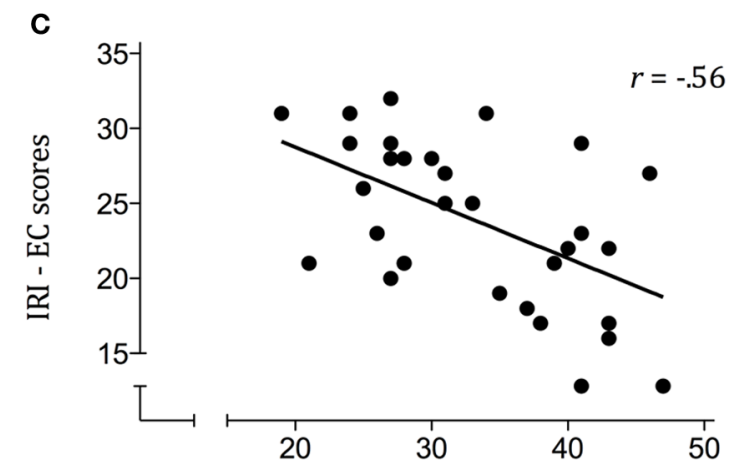

LSRP - PP1 scores

FIGURE 2 | (A) Mean pain ratings for each group indicating an absence of significant difference ( $p=0.35$ ) between LSRP_High and LSRP_Low; (B) Self-reported Empathic Concern (EC) scores significantly differed between LSRP_High and LSRP_Low group; (C) Negative correlation between primary psychopathy (PP1) scores and empathic concern scores. ${ }^{* *} p<0.01$ $p=0.001)$, as well as between the EC subscale and the PP1 subscale $(r=-0.560, p=0.001$; Figure 2C) indicating an inverse relationship between affective empathy and psychopathic traits. Between-group analyses on the pain sensitivity responses indicated no significant differences for the discrimination [PA: $t_{(29)}=$ $0.21, p=0.668$ ] nor the bias scores of the SPQ [B: $t_{(29)}=1.9$, $p=0.184]$.

\section{EEG RESULTS}

\section{General gating effect}

EEG data showed that the maximal change in SG during the visual presentation of the first two stimuli was over the parietal cortex controlateral to the stimulated hand for both experimental groups. As illustrated in Figure 3, subtraction maps (First two pictures - Fixation cross) indicated a strong decrease in left caudal part of the parieto-central region [electrodes 66, 67, 71 in the EGI system (HCGSN) corresponding to P3 in the 10-20 systems] for both groups. A decrease in the $25 \mathrm{~Hz}$ energy band was also found in the same region during the presentation of static stimuli depicting hand in painful and non-painful situations in previous EEG studies using a similar protocol (Voisin et al., 2011a,b,c). Statistical analyses were then restricted to this region specifically showing SG.

In order to assess baseline stability during the Cross Baseline period (-200:0 ms) prior to the first picture onset, a 3 (Conditions: Pain vs. NoPain vs. Neutral) $\times 2$ (Groups: LSRP_Hihg vs. LSRP_Low) repeated measures ANOVA was conducted. No significant effect was observed neither for main effects of Condition $\left[F_{(1,28)}=0.06, p=0.812\right]$ or Group $\left[F_{(1,28)}=1.71, p=0.201\right]$ nor their interaction $\left[F_{(1,28)}=0.31, p=0.583\right]$, reducing the chance that the Cross Baseline period could be the source of subsequent differences.

Figure 4 shows the decrease in the $25 \mathrm{~Hz}$ energy band irrespective of the experimental conditions stabilizing $600-800 \mathrm{~ms}$ after the first picture onset. To investigate this general gating effect, mean energy ratios during Gating period (600:800 ms) and Cross Baseline (-200:0 ms) were compared for each condition using simple $t$-tests against $\mathrm{H}_{0}$ which is the absence of gating $($ ratio $=0)$. On average, modulation amplitude reached.19, corresponding to $19 \%$ of Cross Baseline raw amplitude. Contrasts

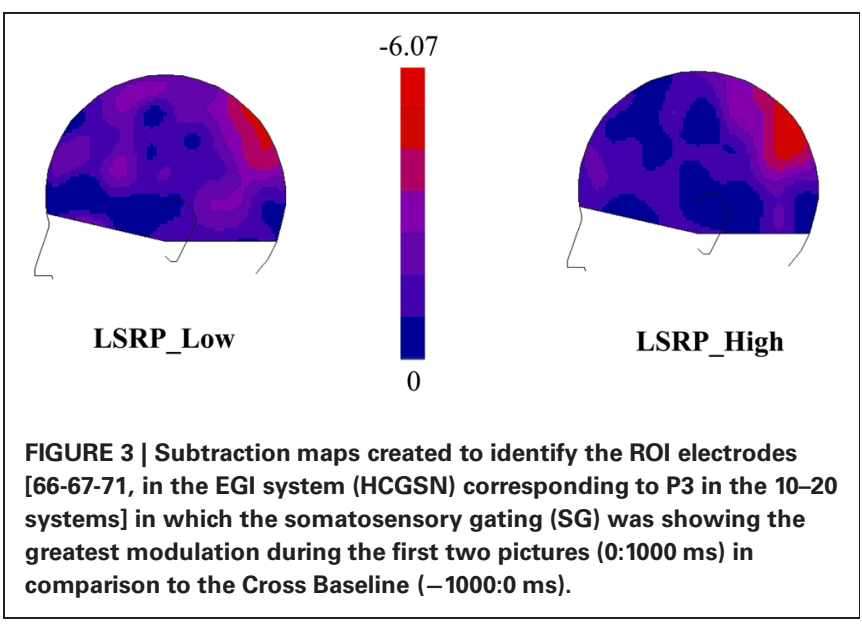




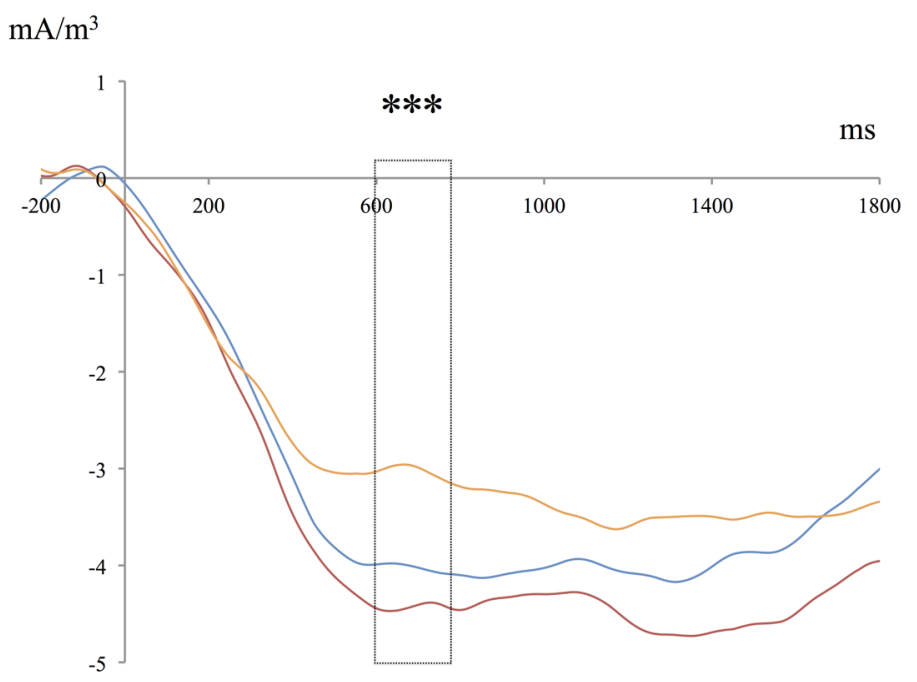

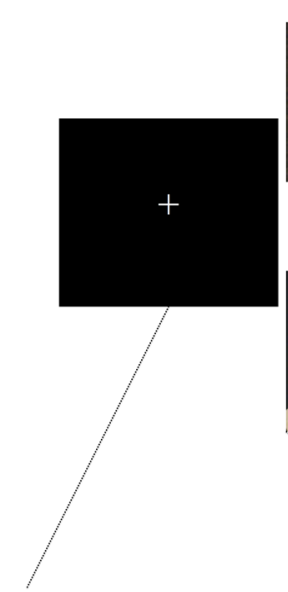

Cross Baseline
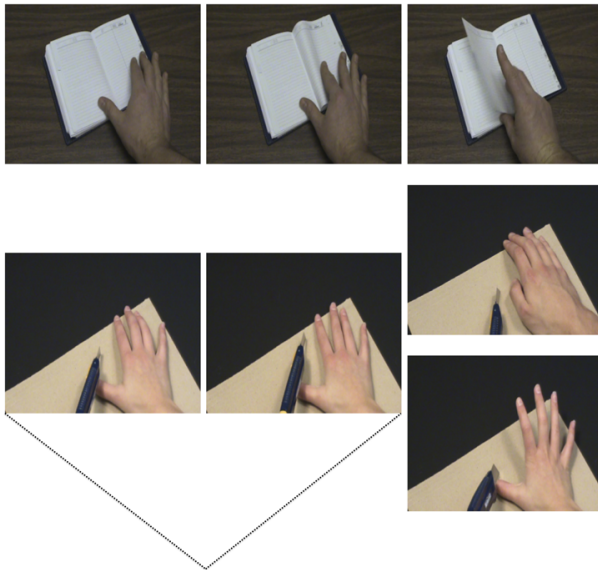

\section{Initial Gating}

FIGURE 4 | Time course of the mean energy $\left(\mathrm{mA} / \mathrm{m}^{3}\right)$ of the somatosensory steady-state response (SSSR) during the presentation of the pseudo-dynamic stimuli. The mean energy of the somatosensory gating (SG) during the first two pictures [initial gating $(600: 800 \mathrm{~ms})$ ] was significantly different from mean energy during the Cross Baseline ( $-200-0 \mathrm{~ms})$ for each condition and for all participants. The magnitude of the SG during the initial gating $(600: 800 \mathrm{~ms})$ was significantly greater in the Pain and NoPain conditions compared to the Neutral condition. ${ }^{* * *} p<0.001$. between Baseline and Gating period were all statistically significant [NoPain: ratio $=0.21 \pm 0.03 ; t_{(29)}=5.9, p<0.001$; Pain: ratio $=0.23 \pm 0.02 ; t_{(29)}=7.8, p<0.001$; Neutral: ratio $=$ $\left.0.14 \pm 0.02 ; t_{(29)}=4.0, p<0.001\right]$, confirming that the observation of the stimuli depicting hands, irrespective of the condition, triggered changes in sensory processing of somatic information in the observer.

\section{Pain anticipation effect}

To assess the possible effect of pain anticipation, mean energy ratios were compared between the three experimental conditions during the Gating period $(600: 800 \mathrm{~ms})$. A significant effect was found for Conditions $\left[F_{(1,28)}=6.8, p=0.014\right]$ but not for Groups $\left[F_{(1,28)}=1.3, p=0.262\right]$; the interaction was not significant $\left[F_{(1,28)}=1.2, p=0.294\right]$. Paired comparisons for
Conditions showed that Neutral significantly differed from Pain $(p=0.013)$ and NoPain $(p=0.043)$ whereas the latter two did $\operatorname{not}(p=0.891)$.

\section{Pain observation effect}

In order to assess Pain Gating, a baseline period was set during the second picture (800:100 ms) for the Pain and NoPain conditions. The stability of this baseline was tested by comparing mean energy for both condition using a 2 (condition: Pain vs. NoPain) $\times 2$ (groups: LSRP Low vs. High) repeated measures ANOVA. No significant effect was observed for the main effects of Condition $\left[F_{(1,28)}=0.27, p=0.612\right]$ or Group $\left[F_{(1,28)}=2.6, p=0.121\right]$ nor their interaction $\left[F_{(1,28)}=0.05, p=0.833\right]$, confirming that Second Picture Baseline would not account for later differences. 
Mean energy ratios were subsequently compared between Pain and NoPain conditions for both groups during the third picture period (1100:1700 ms) through three $(200 \mathrm{~ms})$ time bins (see Figure 5). 2 (condition: Pain vs. NoPain) $\times 2$ (groups: LSRP Low vs. High) repeated measures ANOVA were conducted on the same three time bins. During the $(1100: 1300 \mathrm{~ms})$ period, main effects of Condition $\left[F_{(1,28)}=3.8, p=0.063\right]$ and Group $\left[F_{(1,28)}=\right.$ 2.8, $p=0.114]$ did not reach statistical significance. Still the effect of interaction between both Condition and Group was significant $\left[F_{(1,28)}=4.8, p=0.042\right]$. Post-hoc analyses revealed a significant difference between Pain and NoPain Conditions only for the LSRP_High ( $p=0.014$; LSRP_Low: $p=0.863)$. Throughout the $(1300: 1500 \mathrm{~ms})$ period, no significant effect was observed for main effects of Condition $\left[F_{(1,28)}=2.1, p=0.163\right]$ or Group $\left[F_{(1,28)}=3.5, p=0.074\right]$. However, a significant interaction was found $\left[F_{(1,28)}=6.2, p=0.024\right]$. Post-hoc analyses in each group showed a significant difference between Pain and NoPain Conditions for the LSRP_High group $(p=0.001)$, but not for the LSRP_Low group $(p=0.563)$. For the $(1500: 1700 \mathrm{~ms})$ period, no significant effect was found for main effects of Conditions $\left[F_{(1,28)}=0.8, p=0.382\right]$ or Group $\left[F_{(1,28)}=3.8\right.$, $p=0.074]$ nor their interaction $\left[F_{(1,28)}=3.2, p=0.081\right]$.

\section{CORRELATION BETWEEN THE BEHAVIORAL AND THE EEG RESULTS}

In order to assess the linear dependence between the modulation of SG during pain observation and psychopathic traits, Pearson correlations were used. The analyses performed on the mean energies ratios for the pain picture [Second Picture Baseline (800:100 ms) - Third Picture maximal Gating (1300-1500 ms)/Second Picture Baseline] pointed out some positives associations with LSRP scores. As illustrated in Figure 6, strong positive correlations were found between SG during pain observation and LSRP_Total scores $(r=0.518, p=0.003$; Figure 6A), and PP1 scores ( $r=0.516, p=0.004$; Figure 6B). However, the relationship between SG and the PP2 scores did not reach statistical significance $(r=0.29, p=0.122)$. No significant correlation was found between SSSR and any of the IRI subscales (PT: $r=0.15, p=0.431$; F: $r=-0.06, p=0.763$; EC: $r=-0.21, p=0.284$; D: $r=0.03, p=0.861)$. Finally, no significant relationship was found between SG during pain observation and Pain ratings $(r=0.11, p=0.562)$.

\section{THE INDIRECT EFFECT OF PRIMARY PSYCHOPATHY}

Figure 7 presents the results of the mediation model of direct and indirect effects. The model aimed at testing the interplay between

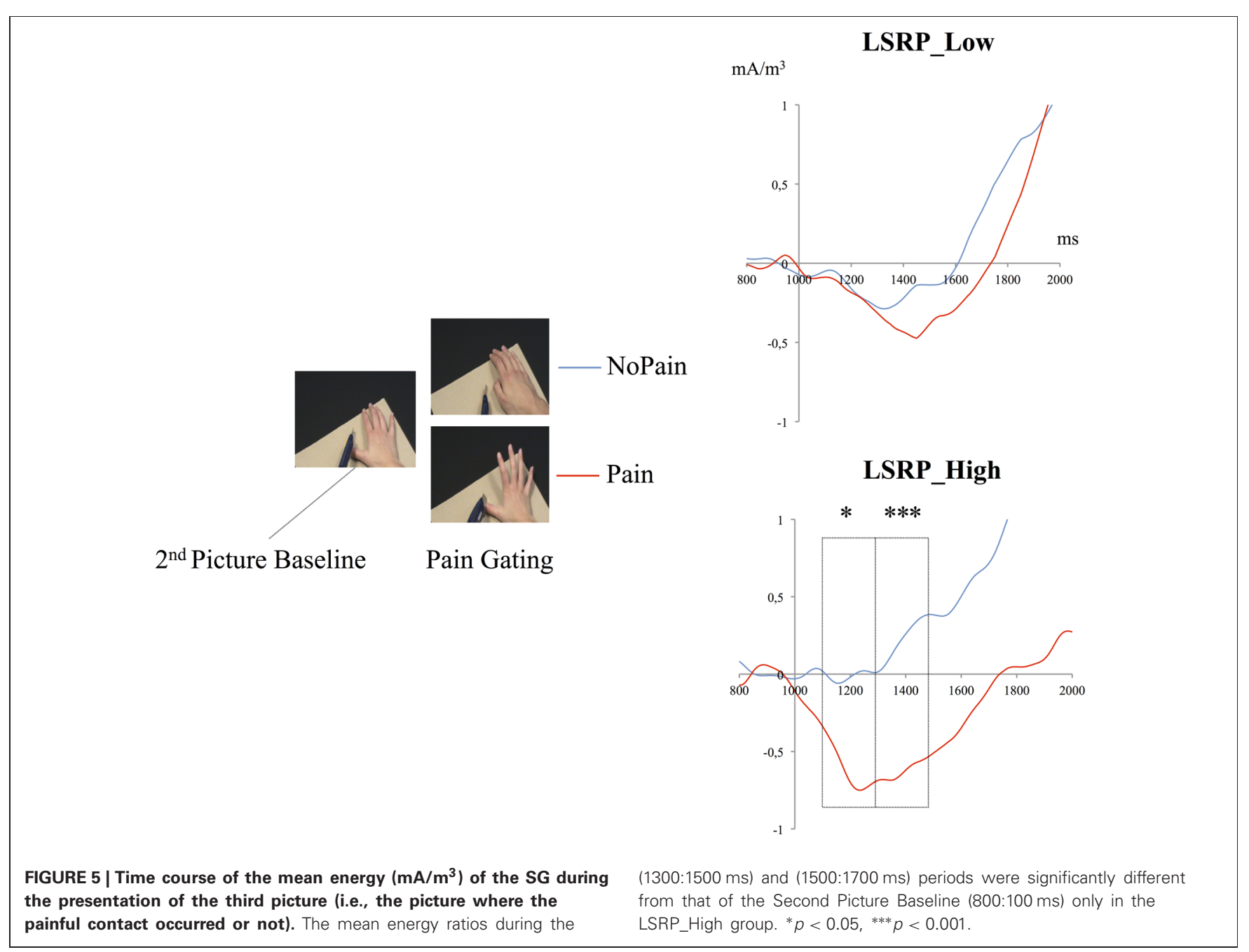




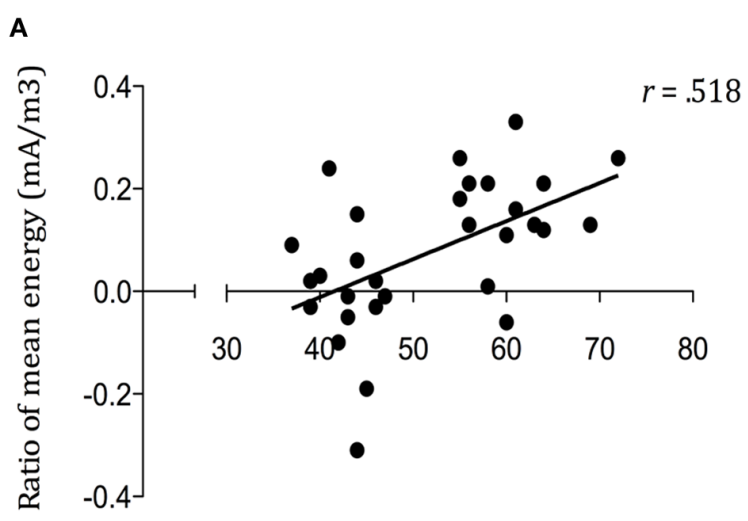

LSRP total scores

B

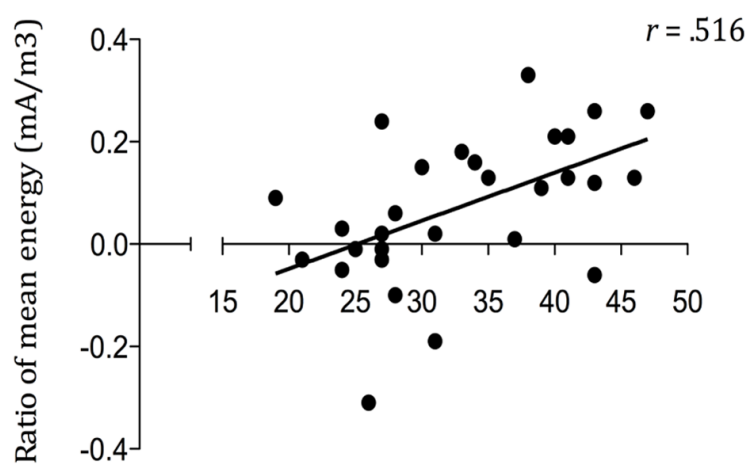

LSRP - PP1 scores

FIGURE 6 | Positive correlations between mean energy $\left(\mathrm{mA} / \mathrm{m}^{3}\right)$ ratios during Pain Gating (1300-1500ms) and (A) self-reported psychopathy total scores $(p=0.003)$; (B) primary psychopathy $(\mathrm{PP} 1)$ subscale scores $(p=0.004)$.

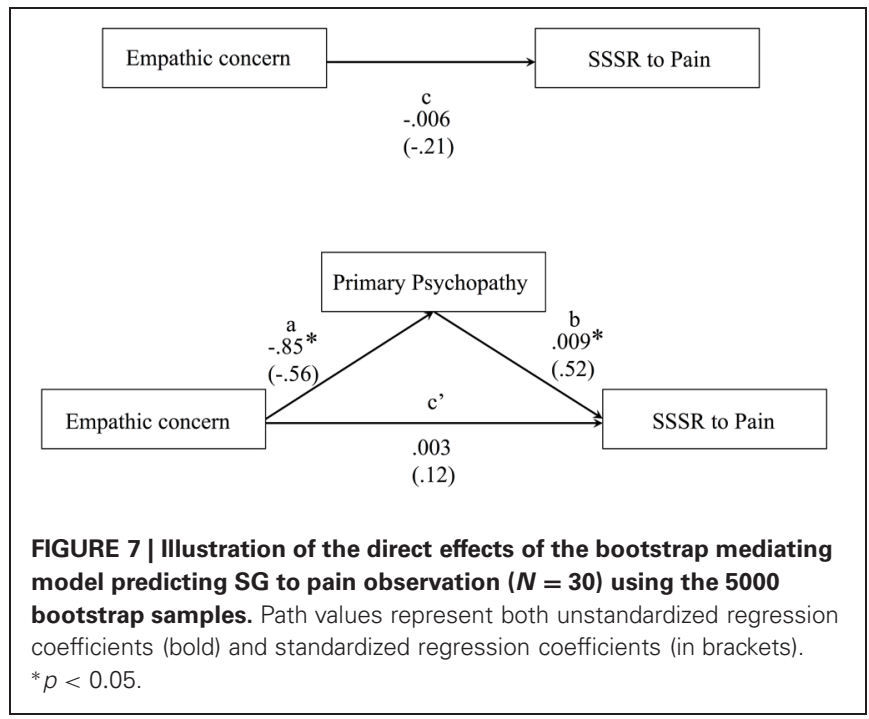

empathy and psychopathy during somatosensory resonance. The results indicated that the total effect of EC on SG to pain (path c) remained non-significant but changed its direction (path c') after introducing primary psychopathy as a mediator. Point-estimate of the indirect effect of EC on SSSR to pain through primary psychopathy was -0.0091 with a $95 \%$ BC confidence interval of -0.0200 to -0.0039 . Because zero was not in the confidence interval, we can conclude that there is a significant indirect effect $\left[\mathrm{R}^{2}\right.$ for the mediating model $\left.=0.277, F_{(2,27)}=5.16, p=0.013\right]$, suggesting that primary psychopathy is a mediator of EC predicting SG to the pain of others. This suggests that psychopathic traits in community individuals contribute to the relation between the affective empathy and somatosensory resonance during pain observation in others.

\section{DISCUSSION}

The goal of this study was to assess changes in somatosensory processing during pain observation in a group of male college students with respect to self-reported empathy and psychopathic traits. Generally, the observation of pseudo-dynamic stimuli depicting hands in Painful and Non-Painful scenarios produced a modulation of the SG response to a mechanical stimulation of the right hand in both high and low psychopathic traits groups. Modulation of the SG was maximal in a parieto-central region contralateral to the stimulated hand. This corroborate previous finding using a similar design (Voisin et al., 2011a) and parallel results showing that observing the body improves tactile performance and modulates SEP (e.g., Taylor-Clarke et al., 2002; Morrison et al., 2007; Cardini et al., 2011). Interestingly, SG specific to pain observation was statistically significant only for the LSRP_High group. Overall, this SG was also positively correlated with affective and interpersonal aspect of psychopathy. Moreover, EC scores were significantly lower in this group compared to LSRP_Low, suggesting that increase somatosensory resonance to other's pain is not exclusively explained by components of affective empathy and may be linked to other personality traits, such as psychopathy. In fact, results from the mediation analysis indicated that primary psychopathy might play a role of mediator in the relation between EC and SSSR to pain.

\section{SELF-REPORTED EMPATHY NEGATIVELY CORRELATED WITH PSYCHOPATHIC TRAITS}

Our behavioral results showed that LSRP_High and Low groups did not differ in their subjective evaluation of pain intensity. This result seems to be in line with previous works reporting that both healthy and conduct disorder adolescents displaying psychopathic traits judged painful stimuli as similarly more painful (Decety et al., 2009) and that pain ratings in juvenile offenders characterized by high and low callous-unemotional traits did not differ (Cheng et al., 2012). The significant difference found between High and Low LSRP groups on IRI-EC subscale adds to the inconsistent findings regarding differences in self-reported empathy among psychopathic and their respective comparison groups. If negative correlations between self-reported empathy and psychopathic traits have been more consistently reported (Sandoval et al., 2000; Jolliffe and Farrington, 2004; Mahmut et al., 2008), 
some studies have failed to show differences on IRI subscales when comparing psychopathic offenders with non-psychopathic offenders with antisocial personality disorder and community samples (Book and Quinsey, 2004; Dolan and Fullam, 2004). Indeed, psychopathy and antisocial personality disorder might be conceived as dimensional constructs (Marcus et al., 2006), hence reducing the possibility to found between group differences on empathy. Besides, the use of self-report empathy with correctional sample may offer limited efficacy as deception, manipulation and grandiose sense of self-worth are at the core of psychopathic manifestation. In the current study, the significant difference found on IRI-EC subscale might be attributed to the composition of the non-forensic sample, as low score on antisocial deviance were found in both groups. The absence of between-group difference in PT is also congruent with current conceptions that psychopathic individuals are seen as having a reduced sensibility to other's distress instead of an incapacity to adopt the psychological perspective of others (Dolan and Fullam, 2004; Blair, 2006).

\section{THE SOMATOSENSORY GATING WAS STRONGER WHEN PAIN WAS ANTICIPATED}

The results of the present study also showed that the increase in the magnitude of SG was more important in the first two pictures for Pain and NoPain conditions compared to Neutral condition. This suggests that contextual dependent effect of the nociceptive elements found in the former conditions might account for the difference in the mean levels of energy. They also support the assumption that whenever our attention is directed to the somatic cause of pain (Bufalari et al., 2007; Lamm et al., 2007), somatosensory processes are engaged by the observer, allowing him or her to create a cerebral representation of others' painful experience by assigning a quantitative sense of pain (Keysers et al., 2010). These results might also be explained by possible pain anticipation. It was previously shown that anticipation of pain in others triggered fear-potentiated startle reflex (Caes et al., 2012) thus potentially modulated the SG to pictures containing nociceptive components. In addition, the study of Caes et al. (2012) demonstrated that startle reflex was blunted in participants depicting higher psychopathic traits. Yet, the current study did not show a significant difference between high and low psychopathic traits group on SG to pain anticipation. The stronger SG found during the first two pictures in which the nociceptive component was displayed compared to neutral pictures indicated a specific change in somatosensory activity during pain anticipation.

\section{PSYCHOPATHIC TRAITS FACILITATED PAIN-RELATED SOMATOSENSORY RESONANCE}

To our knowledge, this is the first study to show that pain observation modulates SG to a greater extend in male college students with high scores on self-reported psychopathy compared to participants with low scores. Other studies have, however, accumulated evidence supporting enhanced somatosensory response to other's pain in male adolescent with high psychopathic traits (Decety et al., 2009; Chen et al., 2012). Specifically, adolescents with conduct disorders and psychopathic traits showed greater sensorimotor resonance for neural response to pain perception compared to healthy adolescents (Decety et al., 2009).
Furthermore, young offenders with high callous-unemotional traits showed stronger $m u$ suppression $(10 \mathrm{~Hz})$ compared to the low ones during pain observation (Cheng et al., 2012). Together, these results are in accordance with our findings, suggesting a greater sensorimotor resonance to other's pain in samples characterized by a reduced capacity for empathy and compassion toward other's distress. This speaks for a more complex link between empathy for pain and resonance than the direct relationship previously proposed, and argue for the contribution of regulation mechanisms allowing prosocial reactions (Decety and Jackson, 2004; Singer et al., 2004; Vachon-Presseau et al., 2012).

Our results also parallel findings from previous studies reporting that SEP elicited by tactile stimulation were modulated by negative emotional stimuli in healthy adults (Montoya and Sitges, 2006) and that the aversion felt during observation of others' pain is negatively correlated with the magnitude of sensorimotor response to others' pain (Avenanti et al., 2009). This is also in line with findings from Decety et al. (2009) who showed greater responses in regions dedicated to affective and sensory components of pain perception in conduct disorders adolescent with psychopathic traits. Specifically, connectivity analysis demonstrated stronger activation of amygdala and striatum together with reduced response in orbitofrontal cortex, suggesting that seeing pain in others did not generate distress in these adolescents but could have led to pleasant feelings. All together, these results suggest that the presence of high psychopathic traits can attenuate the effect of negative emotional arousal caused by the observation of pain in others, thus increasing attention to the sensory components of the stimuli displayed.

Another interesting result consists in the positive correlations found between SG during pain observation and LSRP_Total scores, as well as between SG to pain observation and PP1 subscale scores, which support and extend the findings of Fecteau et al. (2008). As previously demonstrated, participants who scored higher on a specific psychopathic traits subscale (Coldheartedness) showed greater corticospinal inhibition (Fecteau et al., 2008). Interestingly, this subscale measures the absence of deep feeling of guilt and empathy, reflecting the tendency to lack of caring for others (Lilienfeld and Andrews, 1996), all referring to the affective and interpersonal dimension of psychopathy, namely primary psychopathy. However, the negative correlation between empathic concern (IRI-EC) and SG to pain observation did not reach significance. Still, a negative relationship was confirmed between IRI-EC and the PP1 subscale. The fact that the correlations found between the SSSR modulation to pain and both LSRP_total and PP1 subscale are similar (total: $r=0.518$; PP1: $r=0.516$ ) and the absence of significant relation with the PP2 subscale is interesting. These findings suggest that affective and interpersonal aspects of psychopathy constituted the principal factor explaining the modulation of the somatosensory gating. As it might be expected in a community sample study, the PP2 scores resulting from the evaluation of social deviance were low in both groups but still differed significantly; the scores were not comparable to those of incarcerated samples. Nevertheless, results from a community sample indicated that the PP1 factor is more related to high narcissism and prototypical psychopathy compared to the PP2 factor, which tend to be associated with a broad range of personality disorders (Miller et al., 2008). 


\section{PRIMARY PSYCHOPATHY MEDIATED THE LINK BETWEEN EMPATHY AND SOMATOSENSORY RESONANCE}

Results from the Simple Mediator model confirmed the mediating role of primary psychopathy on empathic concern in predicting SG to pain observation. One plausible hypothesis that could account for the absence of significant direct relation between empathic concern and SG to pain observation is the interaction of the suppressor effect revealed by the negative correlation between empathic concern and primary psychopathy with the facilitator effect of primary psychopathy on SG to pain observation. The findings from the mediation analysis could help interpreting the divergent relationship found between enhanced sensorimotor resonance and trait-empathy (Avenanti et al., 2009), as well as between resonance and coldheartedness traits (Fecteau et al., 2008). The results show that psychopathic traits mediated the relation between empathic concern and SG, arguing against the assumption of a straight path between sensorimotor resonance and empathy. This finding is important because it suggests that psychopathic traits in healthy individuals could explain the great inter-individual variability in sensory resonance when decoding pain in others. Further studies will need to dissect the affective and interpersonal qualities that might best contribute to the mediating role of primary psychopathy.

\section{LIMITATIONS AND FURTHER STUDIES}

Some limitations can be pointed out with respect to the proposed interpretation of the findings. First, the use of somatosensory steady-state and time-frequency analysis offer more precision in the frequency domain compared to event-related potential (ERP) and peak to peak analysis but this come with a cost in terms of temporal resolution, as reflected by the use of relatively long time bins (200 ms) in the analyses. Subtle changes in SG relative to temporal dynamics of pain perception might thus have been missed with this method. For instance, the effect of psychopathic traits on pain anticipation was previously shown in a study using ERP with young offenders by assessing early negative arousal (Cheng et al., 2012). Second, the use of extreme scores on the LSRP to form experimental groups may have contributed to the absence of significant SG to pain observation in the LSRP_Low group. Even if this remains speculative, some personality traits and/or emotional factor such as higher negative arousal than individuals in the mid-range of LSRP scores could account for the absence of significant SG during pain observation in the LSRP_Low group. However, mean scores on the PD subscale did not significantly differ between groups and the direction of the relation between negative arousal and sensorimotor response to other's pain needs to be clarified (Meng et al., 2013). Therefore, the present results

\section{REFERENCES}

Aguera, P. E., Jerbi, K., Caclin, A., and Bertrand, O. (2011). ELAN: a software package for analysis and visualization of MEG, EEG, and LFP signals. Comput. Intell. Neurosci. 2011:158970. doi: 10.1155/2011/158970

Avenanti, A., Bueti, D., Galati, G., and Aglioti, S. M. (2005). Transcranial magnetic stimulation highlights the sensorimotor side of empathy for pain. Nat. Neurosci. 8, 955-960. doi: 10.1038/nn1481

Avenanti, A., Paluello, I. M., Bufalari, I., and Aglioti, S. M. (2009). The pain of a model in the personality of an onlooker: influence of state-reactivity and personality traits on embodied

should be interpreted with regards to the direction of the effect instead of its magnitude. Indeed the more robust outcomes, explaining the largest proportion of the variance, were the correlation between LSRP_total/PP1 scores and SG to pain observation suggesting that a dimensional approach might be more appropriate to understand somatosensory resonance with respect to psychopathic traits.

In the current study, the correlation between pain ratings and SG to pain observation was not statistically significant. However, prior studies on pain perception have shown significant positive correlations between sensorimotor processing and evaluations of pain intensity (e.g., Avenanti et al., 2005; Bufalari et al., 2007; Valeriani et al., 2008; Betti et al., 2009). This suggest a multifaceted relationship between sensorimotor resonance and evaluation of others' bodily feelings, suggesting that somatosensory response may not be exclusively related to the intensity of the pain perceived but also to the arousal generated by the stimuli (Bolognini et al., 2013). Future studies will need to clarify the likely interaction of affective arousal on somatosensory processing.

\section{CONCLUSION}

This study demonstrated that observing pain in others triggered somatosensory gating to a greater extends in college male students with high psychopathic traits compared to students with low psychopathic traits. It provides additional evidence on the relationship between personality traits associated with affective and interpersonal dimensions of psychopathy and somatosensory resonance to other's pain. The mediation effect found for psychopathic traits thus gives insight into the complex relationship between trait empathy and somatosensory processing of other's pain. The current study also contribute to extend the growing body of literature on psychopathic correlates in non-incarcerated samples trying to depict a sharper representation of the affectiverelated alterations observed in these individuals, thus supporting a dimensional approach of psychopathy.

\section{ACKNOWLEDGMENTS}

The authors do no report neither relational nor financial conflict of interest. Invaluable technical contributions of Pierre-Olivier Lauzon and Michel-Pierre Coll are acknowledged. Technical support was also provided by the Consortium d'imagerie en neuroscience et santé mentale de Québec (CINQ) for EEG acquisition and analysis. Funding from NSERC, CFI and salary grants from the FRSQ and CIHR to Philip L. Jackson supported this study. This work was made possible thanks to a Leaders Opportunity Fund from the Canadian Foundation for Innovation awarded to Philip L. Jackson.

empathy for pain. Neuroimage 44, 275-283. doi: 10.1016/j.neuro image.2008.08.001

Babiloni, F., Carducci, F., Babiloni, C., and Urbano, A. (1998). Improved realistic Laplacian estimate of highly-sampled EEG potentials by regularization techniques. Electroencephalogr. Clin. Neurophysiol. 106, 336-343.
Baron, R. M., and Kenny, D. A. (1986) The moderator-mediator variable distinction in social psychological research: conceptual, strategic, and statistical considerations. J. Pers. Soc. Psychol. 51, 1173-1182. doi: 10.1037/0022-3514.51.6.1173

Betti, V., Zappasodi, F., Rossini, P. M., Aglioti, S. M., and Tecchio, F. (2009). Synchronous with your 
feelings: sensorimotor \{gamma\} band and empathy for pain. J. Neurosci. 29, 12384-12392. doi: $\quad 10.1523 / J N E U R O S C I .2759-$ 09.2009

Blair, R. J. (2006). "Empathic dysfunction in psychopathic individuals," in Empathy in Mental Illness, 1st Edn., eds T. Farrow and P. Woodruff (New York, NY: Cambridge University Press), 3-16.

Bolognini, N., Rossetti, A., Convento, S., and Vallar, G. (2013). Understanding others' feelings: the role of the right primary somatosensory cortex in encoding the affective valence of others' touch. J. Neurosci. 33, 4201-4205. doi: 10.1523/JNEUROSCI.4498-12.2013

Book, A. S., and Quinsey, V. L. (2004). Psychopaths: cheaters or warriorhawks? Pers. Individ. Dif. 36, 33-45.

Bufalari, I., Aprile, T., Avenanti, A., Di Russo, F., and Aglioti, S. M. (2007). Empathy for pain and touch in the human somatosensory cortex. Cereb. Cortex 17, 2553-2561. doi: 10.1093/cercor/bhl161

Caes, L., Uzieblo, K., Crombez, G., De Ruddere, L., Vervoort, T., and Goubert, L. (2012). Negative emotional responses elicited by the anticipation of pain in others: psychophysiological evidence. J. Pain 13, 467-476. doi: 10.1016/j.jpain.2012.02.003

Cardini, F., Longo, M. R., and Haggard, P. (2011). Vision of the body modulates somatosensory intracortical inhibition. Cereb. Cortex 21, 2014-2022. doi: 10.1093/cercor/bhq267

Chen, C., Yang, C.-Y., and Cheng, Y. (2012). Sensorimotor resonance is an outcome but not a platform to anticipating harm to others. Soc. Neurosci. 7, 578-590. doi: 10.1080/ 17470919.2012.686924

Cheng, Y., Hung, A. Y., and Decety, J. (2012). Dissociation between affective sharing and emotion understanding in juvenile psychopaths. Dev. Psychopathol. 24, 623-636. doi: 10.1017/S095457941200020X

Cheng, Y., Yang, C., Lin, C., Lee, P., and Decety, J. (2008). The perception of pain in others suppresses somatosensory oscillations: a magnetoencephalography study. Neuroimage 40, 1833-1840. doi: 10.1016/j.neuroimage.2008.01.064

Clark, W. C., and Yang, J. C. (1983). "Applications of sensory detection theory to problems in laboratory and clinical pain," in Pain Measurement and Assessment, ed R. Melzack (New York, NY: Raven Press), 15-25.
Cleckley, H. (1941). The Mask of Sanity. St Louis, MO: Mosby.

Danziger, N., Prkachin, K. M., and Willer, J.-C. (2006). Is pain the price of empathy? The perception of others' pain in patients with congenital insensitivity to pain. Brain 129, 2494-2507. doi: 10.1093/brain/awl155

Davis, M. H. (1980). A multidimensional approach to individual differences in empathy. JSAS Cat. Sel. Doc. Psychol. 10, 85.

Decety, J. (2011). Dissecting the neural mechanisms mediating empathy. Emot. Rev. 3, 92-108. doi: $10.1177 / 1754073910374662$

Decety, J., and Jackson, P. L. (2004). The functional architecture of human empathy. Behav. Cogn. Neurosci. Rev. 3, 71-100. doi: $10.1177 / 1534582304267187$

Decety, J., Michalska, K. J., Akitsuki, Y., and Lahey, B. B. (2009). Atypical empathic responses in adolescents with aggressive conduct disorder: a functional MRI investigation. Biol. Psychol. 80, 203-211. doi: 10.1016/j.biopsycho.2008.09.004

Dolan, M., and Fullam, R. (2004). Theory of mind and mentalizing ability in antisocial personality disorders with and without psychopathy. Psychol. Med. 6, 1093-1102.

Fecteau, S., Pascual-Leone, A., and Theoret, H. (2008). Psychopathy and the mirror neuron system: preliminary findings from a non-psychiatric sample. Psychiatry Res. 160, 137-144. doi: 10.1016/j.psychres.2007.08.022

Hall, J. R., Benning, S. D., and Patrick, C. J. (2004). Criterion-related validity of the three-factor model of psychopathy: personality, behavior, and adaptive functioning. Assessment 11, 4-16. doi: 10.1177/1073191103261466

Han, S., Fan, Y., Xu, X., Qin, J., Wu, B., Wang, X., et al. (2009). Empathic neural responses to others' pain are modulated by emotional contexts. Hum. Brain Mapp. 30, 3227-3237. doi: 10.1002/hbm.20742

Hare, R. D. (2003). Hare Psychopathy Checklist-Revised (PCL-R), 2nd Edn. Technical Manual. North Tonawanda, NY: Multi-Health Systems.

Ibáñez, A., Hurtado, E., Lobos, A., Escobar, J., Trujillo, N., Baez, S., et al. (2011). Subliminal presentation of other faces (but not own face) primes behavioral and evoked cortical processing of empathy for pain. Brain Res. 1398, 72-85. doi: 10.1016/j.brainres.2011.05.014

Jackson, P. L., Meltzoff, A. N., and Decety, J. (2005). How do we perceive the pain of others? A window into the neural processes involved in empathy. Neuroimage 24, 771-779. doi: 10.1016/j.neuroimage.2004.09.006

Jackson, P. L., Rainville, P., and Decety, J. (2006). To what do we share the pain of others? Insight from the neural bases of pain empathy. Pain 125, 5-9. doi: 10.1016/j.pain.2006.09.013

Jackson, R. L., Rogers, R., Neumann, C. S., and Lambert, P. L. (2002). Psychopathy in female offenders: an investigation of its underlying dimensions. Crim. Justice Behav. 29, 692-704. doi: $10.1177 / 009385402237922$

Jolliffe, D., and Farrington, D. P. (2004). Empathy and offending. A systematic review and meta-analysis. Aggress. Violent Behav. 9, 441-476. doi: 10.1016/j.avb.2003.03.001

Kernberg, O. (2012a). "Mentalization, mindfulness, insight, empathy, and interpretation," in The Inseparable Nature of Love and Aggression: Clinical and Theoretical Perspectives, ed O. Kernberg (Washington, DC: American Psychiatric Publishing), 57-79.

Kernberg, O. (2012b). Overview and critique of the classification of personality disorders proposed for DSM-V. Swiss Arch. Neurol. Psychiat. 163, 234-238.

Keysers, C., Kaas, J. H., and Gazzola, V. (2010). Somatosensation in social perception. Nat. Rev. Neurosci. 11, 417-428. doi: 10.1038/nrn2833

Kross, E., Berman, M. G., Mischel, W., Smith, E. E., and Wager, T. D. (2012). Social rejection shares somatosensory representations with physical pain. Proc. Natl. Acad. Sci. U.S.A. 108, 6270-6275. doi: 10.1073/pnas. 1102693108

Lamm, C., Decety, J., and Singer, T. (2011). Meta-analytic evidence for common and distinct neural networks associated with directly experienced pain and empathy for pain. Neuroimage 54, 2492-2502. doi: 10.1016/j.neuro image.2010.10.014

Lamm, C., Nusbaum, H. C., Meltzoff, A. N., and Decety, J. (2007). What are you feeling? Using functional magnetic resonance imaging to assess the modulation of sensory and affective responses during empathy for pain. PLOS ONE 12:e1292. doi: 10.1371/journal.pone.0001292

Levenson, M. R., Kiehl, K. A., and Fitzpatrick, C. M. (1995). Assessing psychopathic attributes in a noninstitutionalized population. J. Pers. Soc. Psychol. 68, 151-158. doi: 10.1037/0022-3514.68.1.151
Lilienfeld, S. O., and Andrews, B. P. (1996). Development and preliminary validation of a self-report measure of psychopathic personality traits in noncriminal populations. J. Pers. Assess. 66, 488-524. doi: 10.1207/s15327752jpa6603_3

Mahmut, M. K., Homewood, J., and Stevenson, R. J. (2008). The characteristics of non- criminals high in psychopathic traits: are they similar to criminal psychopaths? J. Res. Pers. 42, 679-692. doi: 10.1016/j.jrp.2007.09.002

Marcus, D. K., Lilienfeld, S. O., Edens, J. F., and Poythress, N. G. (2006). Is antisocial personality disorder continuous or categorical? A taxometric analysis. Psychol. Med. 36, 1571-1582. doi: 10.1017/S0033291706008245

Martínez-Jauand, M., GonzálezRoldán, A. M., Muñoz, M. A., Sitges, C., Cifre, I., and Montoya, P. (2012) Somatosensory activity modulation during observation of other's pain and touch. Brain Res. 1467, 48-55. doi: 10.1016/j.brainres.2012. 05.055

Mayer, A. R., Hanlon, F. M., Franco, A. R., Teshiba, T. M., Thoma, R. J., Clark, V. P., et al. (2009). The neural networks underlying sensory gating. Neuroimage 44, 182-189. doi: 10.1016/j.neuroimage.2008.08.025

Meng, J., Jackson, T., Chen, H., Yang, L. H., Su, Y., and Huang, X. (2013). Pain perception in the self and observation of others: an ERP investigation. Neuroimage 72, 164-173. doi: 10.1016/j.neuroimage.2013.01.024

Miller, J. D., Gaughan, E. T., and Pryor, L. R. (2008). The Levenson SelfReport Psychopathy Scale: an examination of the personality traits and disorders associated with the LSRP factors. Assessment 15, 450-463. doi: $10.1177 / 1073191108316888$

Montoya, P., and Sitges, C. (2006). Affective modulation of somatosensory-evoked potentials elicited by tactile stimulation. Brain Res. 1068, 205-212. doi: 10.1016/j.brainres.2005.11.019

Morrison, I., Lloyd, D., di Pellegrino, G., and Roberts, N. (2004). Vicarious responses to pain in anterior cingulate cortex is empathy a multi-sensory issue? Cogn. Aff. Behav. Neurosci. 4, 270-278.

Morrison, I., Poliakoff, E., Gordon, L., and Downing, P. E. (2007). Response-specific effects of pain observation on motor behavior. Cognition 104, 407-416. doi: 10.1016/j.cognition.2006.07.006

Perrin, F., Pernier, J., Bertrand, O., and Echallier, J. F. (1989). 
Spherical splines for scalp potential and current density mapping. Electroencephalogr. Clin. Neurophysiol. 72, 184-187. doi: 10.1016/0013-4694(89)90180-6

Poythress, N., and Skeem, J. (2006). "Disaggregating psychopathy: where and how to look for variants," in the Handbook of psychopathy, ed C. Patrick (New York, NY: Guilford Press), 172-192.

Preacher, K. J., and Hayes, A. F. (2004). SPSS and SAS procedures for estimating indirect effects in simple mediation models. Behav. Res. Methods Instrum. Comput. 36, 717-731.

Preacher, K. J., and Hayes, A. F. (2008). Asymptotic and resampling strategies for assessing and comparing indirect effects in multiple mediator models. Behav. Res. Methods 40, 879-891.

Saarela, M. V., Hlushchuk, Y., Williams, A. C., Schurmann, M., Kalso, E., and Hari, R. (2007). The compassionate brain: humans detect pain intensity from another's face. Cereb. Cortex 17, 230-237. doi: 10.1093/cercor/bhj141

Salekin, R. T., Rogers, R., and Sewell, K. W. (1997). Construct validity of psychopathy in a female offender sample: a multivariatemultimethod evaluation. J. Abnorm.
Psychol. 106, 576-585. doi: 10.1037/0021-843X.106.4.576

Sandoval, A. R., Hancock, D., Poythress, N. G., Edens, J. F., and Lilienfeld, S. O. (2000). Construct validity of the psychopathic personality inventory in a correctional sample. J. Pers. Assess. 74, 262-281. doi: 10.1207/S1532775 2JPA7402_7

Singer, T., Seymour, B., O’Doherty, J., Kaube, H., Dolan, R. J., and Frith, C. D. (2004). Empathy for pain involves the affective but not sensory components of pain. Science 303, 1157-1162. doi: 10.1126/science. 1093535

Tallon-Baudry, C., and Bertrand, O. (1999). Oscillatory gamma activity in humans and its role in object representation. Trends Cogn. Sci. 3, 151-162. doi: 10.1016/S13646613(99)01299-1

Taylor-Clarke, M., Kennett, S., and Haggard, P. (2002). Vision modulates somatosensory cortical processing. Curr. Biol. 12, 233-236. doi: 10.1016/S0960-9822(01)00681-9

Vachon-Presseau, E., Martel, M. O., Roy, M., Caron, E., Jackson, P. L., and Rainville, P. (2011). The multilevel organization of vicarious pain responses: effects of pain cues and empathy traits on spinal nociception and acute pain. Pain 152, 1525-1531. doi: 10.1016/j.pain.2011.02.039

Vachon-Presseau, E., Roy, M., Martel, M. O., Albouy, G., Chen, J., Budell, L., et al. (2012). Neural processing of sensory and emotional-communicative information associated with the perception of vicarious pain. Neuroimage 63, 54-62. doi: 10.1016/j.neuroimage.2012.06.030

Valeriani, M., Betti, V., Le Pera, D., De Armas, L., Miliucci, R., Restuccia, D., et al. (2008). Seeing the pain of others while being in pain: a laser-evoked potentials study. Neuroimage 40, 1419-1428. doi: 10.1016/j.neuroimage.2007.12.056

Voisin, J. I. A., Marcoux, L.-A., Canizales, D. L., Mercier, C., and Jackson, P. L. (2011a). I am touched by your pain: limb-specific modulation of the cortical response to a tactile stimulation during pain observation. J. Pain 12, 1182-1189. doi: 10.1016/j.jpain.2011.06.005

Voisin, J. I. A., Rodrigues, E., Hétu, S., Jackson, P., Vargas, C., Malouin, F., et al. (2011b). Modulation of the response to a somatosensory stimulation of the hand during the observation of manual actions. Exp. Brain Res. 208, 11-19. doi: 10.1007/s00221-0102448-3
Voisin, J. I. A., Mercier, C., Jackson, P. L., Richards, C. L., and Malouin, F. (2011c). Is somatosensory excitability more affected by the perspective or modality content of motor imagery? Neurosci. Lett. 493, 33-37. doi: 10.1016/j.neulet.2011.02.015

Conflict of Interest Statement: The authors declare that the research was conducted in the absence of any commercial or financial relationships that could be construed as a potential conflict of interest.

Received: 08 March 2013; accepted: 26 May 2013; published online: 19 June 2013.

Citation: Marcoux L-A, Michon P-E, Voisin IIA, Lemelin S, Vachon-Presseau $E$ and Jackson PL (2013) The modulation of somatosensory resonance by psychopathic traits and empathy. Front. Hum. Neurosci. 7:274. doi: 10.3389/ fnhum.2013.00274

Copyright (c) 2013 Marcoux, Michon, Voisin, Lemelin, Vachon-Presseau and Jackson. This is an open-access article distributed under the terms of the Creative Commons Attribution License, which permits use, distribution and reproduction in other forums, provided the original authors and source are credited and subject to any copyright notices concerning any third-party graphics etc. 